$$
\text { NASA - CR - 183,252 }
$$

NASA-CR-183252

19880021195

PREPRINT SERIES

No 203

RADIO AND INFRARED PROPERTIES OF YOUNG STARS

Nino Panagia

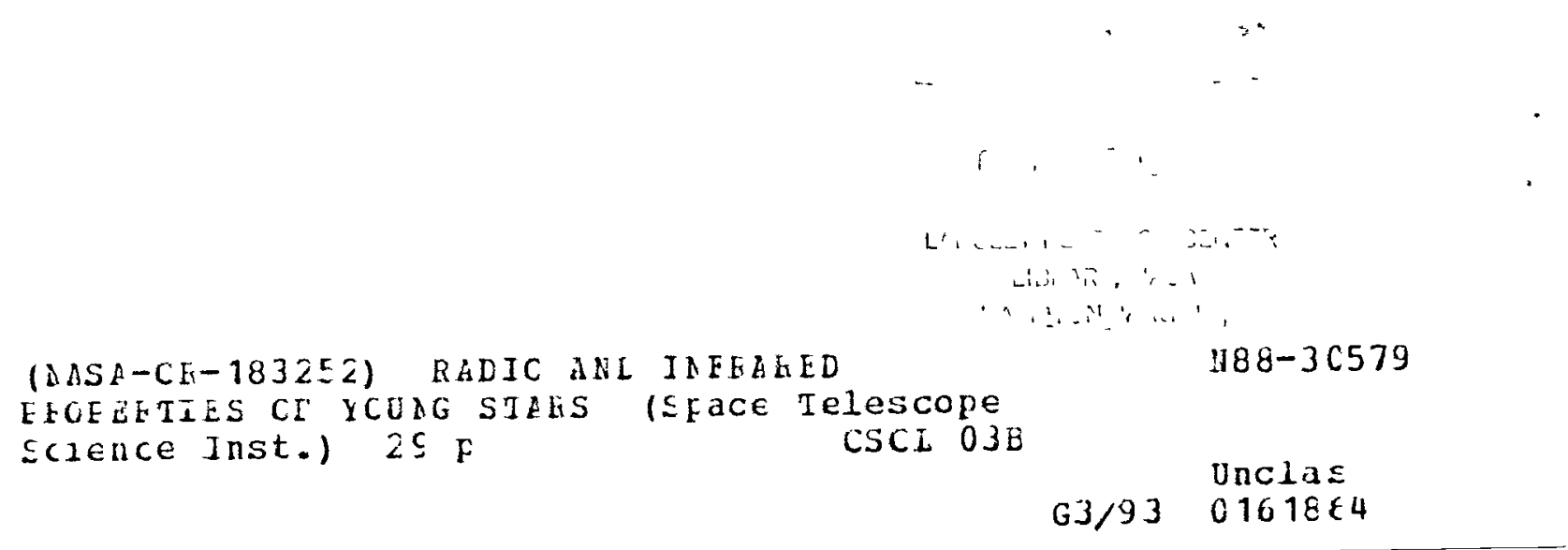

September 1987

SPACE TELESCOPE SCIENCE INSTITUTE

3700 San Martın Drive Baltımore, MD 21218 


\title{
RADIO AND INFRARED PROPERTIES OF YOUNG STARS
}

\author{
Nino Panagıa* \\ Space Telescope Science Institute \\ Baltimore, MD, USA, \\ and \\ University of Catania, Italy
}
Invited Lecture given at the NATO ASI
"Galactic and Extragalactic Star Formation"
June 21 - July 4, 1987
Whistler, B.C., Canada

* Affiliated to the Astrophysıcs Divisıon, Space Sciences Department of ESA. 


\begin{abstract}
The observations and the theory of the radio and infrared emission from pre-mainsequence stars are reviewed and discussed.
\end{abstract}

\title{
1. INTRODUCTION
}

Observing young stars or, more appropriatedly, pre-main-sequence (PMS) stars, in the infrared and in the radio has the advantage over optical observations that the heavy extinction associated with a star forming region is only a minor problem, if at all, so that the whole region can be studied thoroughly. Therefore, it means being able: i) to search for stars and do statistical studies on the rate of star formation, ii) to determine their luminosity, hence, to study luminosity functions and initial mass functions down to low masses, iii) to study their spectra and, thus, to determine the prevailing conditions at and near the surface of a newly born star and its relations with the surrounding environment This third point is the main interest of my review. In fact, I shall limit myself to considering the observations concerning the processes of outflows from, and accretion onto, PMS stars and the theory necessary to interpret them. Section 2 will discuss the radiative processes relevant in stellar outflows. The main observational results will be presented in Section 3. A discussion of the statistical properties of stellar winds from PMS stars will be given in section 4.

\section{EMISSION FROM EXTENDED OUTFLOWS: A THEORETICAL OVERVIEW}

In this section we shall review the problem of radiation transfer applied to the case of stellar outflows. Quite of number of authors have contributed to this field, both for the formation of the continuum (Panagia and Felli 1975, Wright and Barlow 1975, Olnon 1975, Marsh 1975, Chiuderi and Torricelli Ciamponi 1978, Felli and Panagia 1981, Schmid-Burgk 1982, Castor and Simon 1983, Lamers and Waters 1984, Reynolds 1986, Felli and Panagia 1987) and the lines (Krolik and Smith 1981, Sımon et al. 1983, Rodriguez and Cantó 1983, Fell et al. 1985a, Hamann and Simon 1986, Panagia et al. 1987). The most important points and results are outlined and discussed in the following subsections.

\section{Assumptions - Basic Transfer Problem}

In order to make the transfer problem easily tractable the following assumptions are usually made (e.g., Panagia and Felli 1975):

1. The wind is spherically symmetric with a steady mass loss, $\dot{M}$.

2. The wind is fully ionized. This condition, combined with the continuity equation gives the behaviour of the electron density $n_{e}$ with the distance, $r$, from the center of the star

$$
n_{e}=\frac{\dot{M}}{4 \pi r^{2} v(r) \mu_{e} m_{H}}
$$

where $v(r)$ is the wind velocity, $\mu_{e}$ is the mean atomic weight per electron

$$
\mu_{e}=\frac{\sum_{\imath} X_{2} m_{\imath}}{\sum_{i} X_{2} Z_{2}}
$$




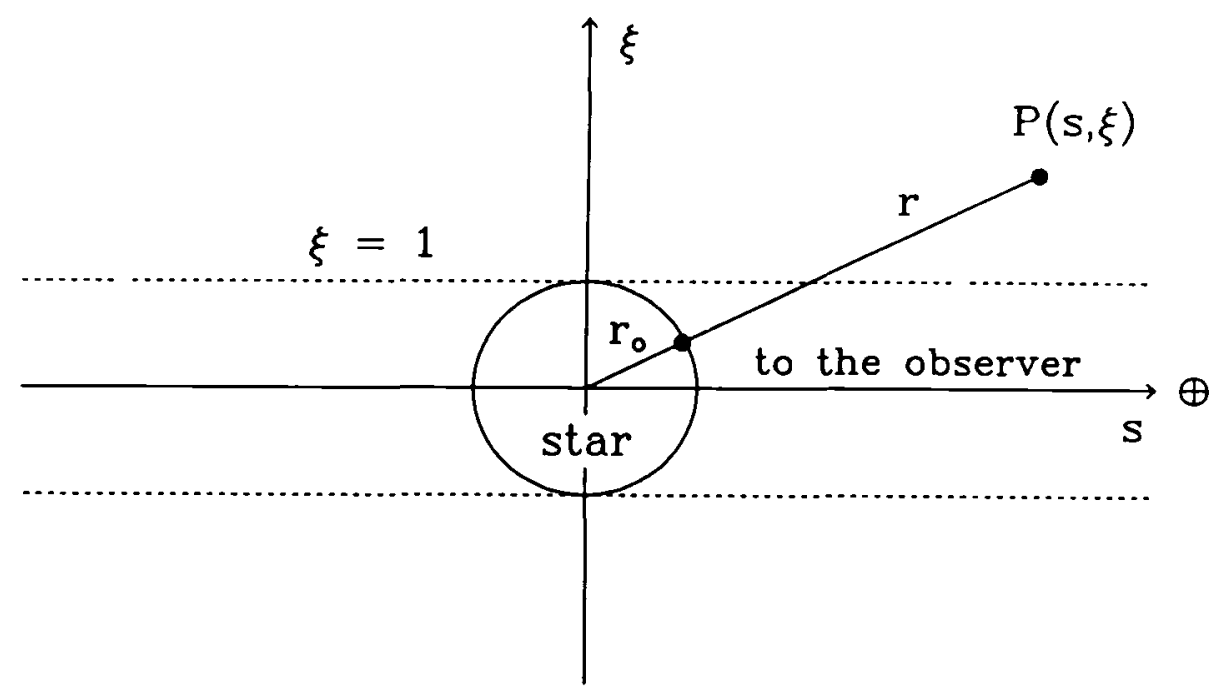

Fig. 1 - The geometry of the star+wind system.

with $X_{2}, m_{1}$ and $Z_{2}$ being the abundance by number, the mass and the charge of the i-th ion, and $m_{H}$ is mass of a hydrogen atom.

3. The wind velocity increases with radius like a power law

$$
v=v_{o}\left(r / r_{o}\right)^{\gamma}
$$

where $v_{o}$ and $r_{o}$ are the initial velocity and radius, respectively, unt 11 it reaches its terminal value $v_{\infty}$ at a radius $r_{1}$

$$
v=v_{\infty} \quad r \geq r_{1}=r_{o}\left(v_{\infty} / v_{o}\right)^{1 / \gamma}
$$

4. The wind is isothermal at the same temperature as the stellar photosphere, $\mathrm{i}$ e.,

$$
T_{\text {wind }}=T_{\text {star }}
$$
valid

5. The wind material is in quasi-LTE conditions, in the sense that the Kirchoff law is

$$
j_{\nu}=\kappa_{\nu} B_{\nu}
$$

where $j_{\nu}$ and $\kappa_{\nu}$ are the emissivity and the absorption coefficient, respectively. With these assumptions the radiative transfer equation along any line of sight can be written as

$$
\frac{d I}{d s}=-\kappa I+\jmath
$$

where $s$ is the current coordinate along the line of sight (see Figure 1 which illustrates the geometry of the problem). Its solution is

$$
I=I_{o} e^{-\tau}+B\left(1-e^{-\tau}\right)
$$

where $I_{o}$ is the background intensity and $\tau$ is the optical depth as integrated along the line of sight, i.e.

$$
\tau=2 \int_{0}^{\infty} \kappa d s
$$


With our assumptions it is possible to express the total optical along any line of sight (assuming the opacity to be proportional to $n_{e}^{2}$ ) as

$$
\tau=\tau_{o} \xi^{-(3+2 \gamma)}
$$

where $\tau_{o}$ represents the optical depth for impact parameter equal to unity, i.e., for the line of sight tangent to the stellar disk.

The radiation that we receive from the system star+wind is the intensity integrated over all lines of sight parallel to line joining us to center of the star. It is immediately clear that for $\xi<1$, i.e., for lines of sight which project on the stellar disk,

$$
\begin{aligned}
& I_{o}=B \\
& I=B e^{-\tau}+B\left(1-e^{-\tau}\right)=B \quad \xi<1
\end{aligned}
$$

In other words the presence of a wind in front of the star does not affect the radiation we receive from the stellar disk because the amount of radiation which is absorbed is perfectly compensated by the emission of the wind.

For impact parameters greater than the stellar radius there is no background radiation so that we have simply

$$
I=B\left(1-e^{-\tau}\right) \quad \xi \geq 1
$$

The total emission received from the star+wind system will then be given by

$$
F_{\nu}=\int I_{\nu} d \Omega=\frac{1}{4 \pi d^{2}} 4 \pi R^{2} \pi B_{\nu}\left[1+2 \int_{1}^{\infty}\left(1-e^{-\tau}\right) \xi d \xi\right]
$$

Equation (12) can be rewritten as

$$
F_{\nu}=\frac{4 \pi R^{2}}{4 \pi d^{2}} \pi B_{\nu}[1+\epsilon]
$$

where $R$ is the photospheric radius and $d$ is the distance; $\epsilon$ represents the excess of radiation due to the presence of the wind and is given by

$$
\epsilon=2 \int_{1}^{\infty}\left(1-e^{-\tau}\right) \xi d \xi
$$

Equations (14) and (15) are the basic transfer equations which govern both for the continuum and the emission line problems. Analytical solutions to eq. (15) can be obtained for two cases, which eventually turn out to cover all relevant possibilities (Panagia and Felli 1987):

i. The quasi-optically case, which is valid for moderate optical depths $\left(\tau_{o} \lesssim 3\right)$. In this case by expanding equation (15) to second order terms and after some manipulation one obtains

$$
\epsilon=\frac{a \tau_{o}}{1+b \tau_{o}}
$$

where the coefficients $a$ and $b$ are simple functions of the velocity slope $\gamma$ :

$$
a=\frac{2}{1+2 \gamma} \quad b=\frac{1+2 \gamma}{8+8 \gamma}
$$


Table 1

Coefficients of the continuous emission from the wind

\begin{tabular}{ccccccc}
\hline \hline & $a$ & $b$ & $c$ & $d$ & $J(\gamma)$ & $\alpha$ \\
\hline 0.0 & 2.000 & 0.125 & 2.679 & 0667 & 0.785 & 0.600 \\
0.5 & 1.000 & 0.167 & 1.772 & 0.500 & 0.667 & 0.950 \\
1.0 & 0.667 & 0.188 & 1.489 & 0.400 & 0.589 & 1.160 \\
2.0 & 0.400 & 0.208 & 1.276 & 0.286 & 0.491 & 1.400 \\
\hline
\end{tabular}

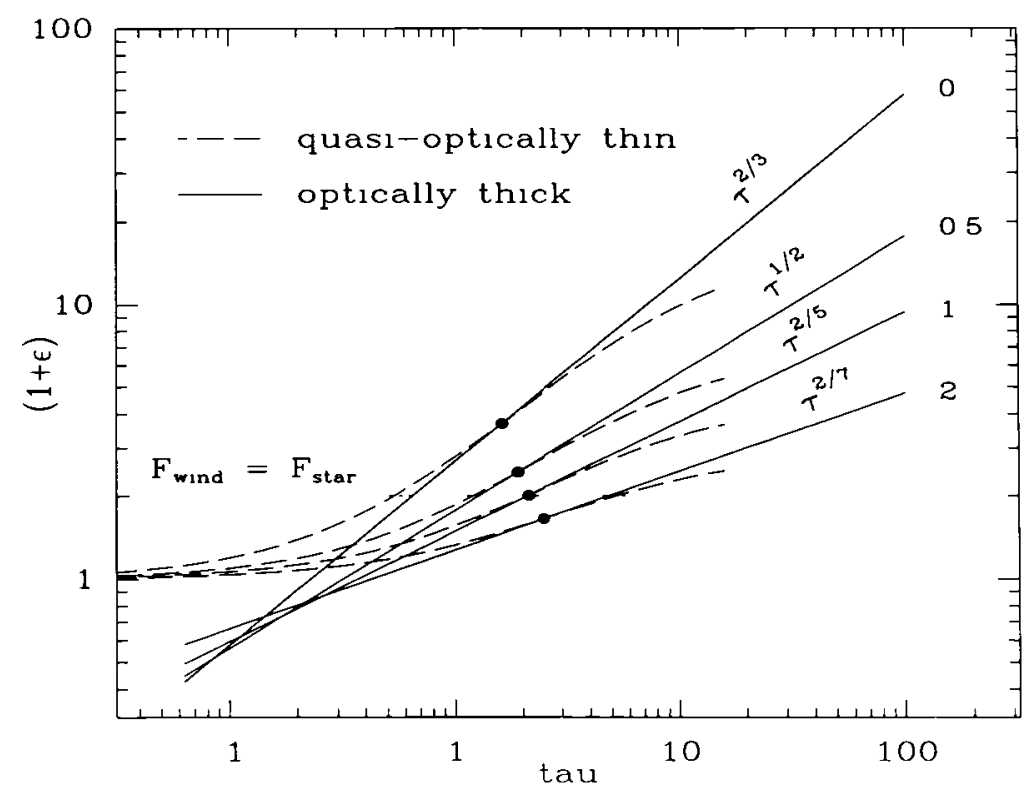

Fig. 2 - The quantity $(1+\epsilon)$ as a function of the optical depth: curves for both the quasi-optically thin (dashed lines) and the optically thick (solid lines) cases are shown for some values of $\gamma$. The points where the two approximations meet are marked as well as the line of equal flux contributed by the wind as emitted by the star.

Values of $a$ and $b$ for some values of $\gamma$ are given in Table 1. Since in general $a \gg b$ and $b \ll 1$ the radiation excess $\epsilon$ increases essentially linearly with the optical depth and only for relatively high values of $\tau$ (say $>1 / 2$ ) the rise departs appreciably from linearity.

ii. The optically thick case: here the optical depth is large $\left(\tau_{0}>3\right)$ and therefore one can write

$$
1+\epsilon=1+2 \int_{1}^{\infty}\left\{1-\exp \left[-\tau_{o} \xi^{-(3+2 \gamma)}\right]\right\} \xi d \xi \simeq \int_{0}^{\infty}\left\{1-\exp \left[-\tau_{o} \xi^{-(3+2 \gamma)}\right]\right\} \xi d \xi
$$

This equation correspond to a gamma function, so that we obtain:

$$
1+\epsilon=\Gamma\left(1-\frac{2}{2 \gamma+3}\right) \tau_{o}^{\frac{2}{2 \gamma+3}}=c \tau_{o}^{d}
$$

Table 1 reports values of the coefficients $c=\Gamma[1-2 /(2 \gamma+3)]$ and $d=2 /(2 \gamma+3)$ for some values of $\gamma$. It is apparent that in this case the increase of the radiation excess with the optical depth is systematically slower than linear. 
If we plot the quantity $(1+\epsilon)$ as computed with eq. (16) and (19), respectively, we see (Fig. 2) that the two curves join quite smoothly with each other for optical depths around 2. Detailed numerical calculations show that where the two curves intersect each other the analytical formulae give the correct value of $\epsilon$ to within better than $1.7 \%$. Therefore, one can safely use eq. (16) for lower values of the optical depth and eq. (19) for higher values, thus covering the whole possible range with two simple formulae. Finally, it is worth noting that the excess radiation becomes equal to the original stellar emission for values of the optical depth of the order of unity: in particular 0.53, 2.09 and 4.82 for gamma values of 0,1 and 2 , respectively (cf fig. 2).

\subsection{The continuum}

The main emission mechanism in the infrared and in the radio is the free-free (or bremsstrahlung) radiation ( $f-f)$. The free bound transitions (i.e. recombinations of free electrons to bound levels), which dominate the optical emission, are of modest importance in the near infrared (they provide $20 \%$ of the continuum at $2.2 \mu \mathrm{m}$ ) and become completely negligible at longer wavelengths. Therefore, for the sake of simplicity in the following discussion we shall consider only the f-f process. The f-f opacity per unit length, which we express as the product of the electron and ionic densities times a function $k_{\nu}$, is given by

$$
\kappa_{\nu}=n_{e} n_{2} k_{\nu}=3.69 \times 10^{8} n_{e} n_{\imath}\left[1-e^{-\frac{h v}{k T}}\right] g_{f f} T^{-1 / 2} \nu^{3}
$$

At radio frequencies $k_{\nu}$ can be approximated quite accurately as (Mezger and Henderson, 1967)

$$
k_{\nu}=8.44 \times 10^{-28}\left[\frac{\nu}{10 \mathrm{GHz}}\right]^{-21}\left[\frac{T}{10^{4} K}\right]^{-135}
$$

Although this formula is accurate only in the radio domain, for qualitative discussions it can also be used advantageously at infrared wavelengths as well. Inserting eq. (20) into (10) the optical depth at impact parameter equal to unity is evaluated to be

$$
\tau_{o}=2 J(\gamma) n_{o}^{2} R k_{\nu} \propto \frac{\dot{M}^{2}}{R^{3} v_{o}^{2}} \nu^{-21} T^{-135}
$$

with $J(\gamma)$ defined as

$$
J(\gamma)=\frac{\sqrt{\pi}}{2} \frac{\Gamma\left(\gamma+\frac{3}{2}\right)}{\Gamma(\gamma+2)}
$$

Values of $J(\gamma)$ for some values of $\gamma$ are given in Table 1 . It is straightforward now to estimate the emitted flux by using equations (14), (16) and (19). In the quasi-optically thin case the flux is virtually proportional to $\tau_{o}$ and therefore the main functional dependences of the excess flux are

$$
F_{w \imath n d}=\frac{4 \pi R^{2}}{4 \pi d^{2}} \pi B_{\nu} \epsilon \propto \frac{R^{2}}{d^{2}} B_{\nu} \tau_{o} \propto \frac{\dot{M}^{2}}{v_{o}^{2}} R^{-1} T^{-135} \nu^{-21} B_{\nu}
$$

On the other hand, in the optically thick case the total flux is

$$
F_{t o t a l}=\frac{4 \pi R^{2}}{4 \pi d^{2}} \pi B_{\nu}(1+\epsilon) \propto \frac{R^{2}}{d^{2}} B_{\nu} \tau_{o} \frac{2}{2 \gamma+3}
$$


which, expanding the dependences of $\tau_{0}$ upon the various parameters, becomes:

$$
F_{\nu} \propto d^{-2} R^{\frac{4 \gamma}{2 \gamma+3}} T^{\frac{2 \gamma+03}{2 \gamma+3}} \nu^{\frac{4 \gamma+18}{2 \gamma+3}}\left(\frac{\dot{M}}{v_{0}}\right)^{\frac{4}{2 \gamma+3}}
$$

The first important point is the frequency dependence which correspond to a power law whose spectral index $\alpha\left(F_{\nu} \propto \nu^{\alpha}\right)$ is given by

$$
\alpha=\frac{4 \gamma+1.8}{2 \gamma+3}
$$

i.e., always lower than the black body slope of 2 (see Table 1). This is because at lower frequency the opacity is higher (cf. eq. (21)) and, therefore, the radius at which the optical depth is of the order of unity becomes larger and the effective emitting surface increases. In other words, the emission correspond to that of a black body (as expected for a well behaved, optically thick, thermal source) whose size increases with wavelength, so that the overall decrease of flux with wavelength is slower than it is for a Planck curve. In fact, we can define an effective radius to be that of a black body at the wind temperature which emits the same flux as the wind. Therefore, from eq. (14) we obtain

$$
R_{e f f} \simeq \sqrt{1+\epsilon} R
$$

Therefore, for large $\tau_{o}$ the effective radius becomes a simple function of the frequency:

$$
R_{e f f} \propto \nu^{-\frac{42}{2 \gamma+3}}
$$

Such an effective radius can be considerably larger than the photospheric radius. For example, in the radio the size of the emitting radius can be several thousands of stellar radii for typical values of the mass loss. Therefore, since the accelerating force becomes weak at large radii, the velocity in the radio emitting region can be expected to be essentially constant. In the constant velocity case $(\gamma=0)$ one recovers the "classical" formulae of the radio emission from winds (Panagia and Felli 1975)

$$
\begin{aligned}
F_{\nu}= & 512\left[\frac{\nu}{10 \mathrm{GHz}}\right]^{06}\left[\frac{T}{10^{4} K}\right]^{01}\left[\frac{\dot{M}}{10^{-6} \frac{M_{\mathrm{O}}}{\mathrm{yr}}}\right]^{4 / 3}\left[\frac{v_{\infty}}{100 \mathrm{~km} \mathrm{~s}^{-1}}\right]^{-4 / 3} \times \\
R_{\nu}= & {\left[\frac{d}{1 \mathrm{kpc}}\right]^{-2} \mathrm{~m} \mathrm{Jy} } \\
& {\left[\frac{\dot{M}}{10^{-6} \frac{M_{\odot}}{\mathrm{yI}}}\right]^{14}\left[\frac{\nu}{10 \mathrm{GHz}}\right]^{-0.7}\left[\frac{T}{10^{4} K}\right]^{-045} } \\
\theta= & 0.042\left[\frac{\nu}{10 \mathrm{GHz}}\right]^{-07 / 3}\left[\frac{T}{10^{4} K}\right]^{-045}\left[\frac{\dot{\mathrm{km}}}{10^{-6} \frac{M_{\mathrm{O}}}{\mathrm{yr}}}\right]^{2 / 3} \\
& {\left[\frac{v_{\infty}}{100 \mathrm{~km} \mathrm{~s}^{-1}}\right]^{-2 / 3}\left[\frac{d}{1 \mathrm{kpc}}\right]^{-1} \operatorname{arcsec} }
\end{aligned}
$$


In this case a mass losing star is expected to be detected as having a characteristic $\nu^{06}$ radio spectrum and a source size of few hundrendths of an arcsec. We note that the theory is quite simple and the radio flux depends in a simple manner on $\dot{M}$ Therefore, radio measurements can provide reliable determinations of the mass loss rate provided that the terminal velocity can be estimated independently. Even so, to do it right one has to make sure that the radio spectrum is really that of a wind, i.e., that the spectral index is $\sim 0.6$ indeed. As discussed by Felli and Panagia (1981) this requires making radio measurements at three widely spaced frequencies.

\subsection{The emission lines}

The problem of line radiation transfer is much more complicate because it involves bound levels which may not be in any simple equilibrium and because by its very nature the line opacity is a rapidly varying function of the frequency. However, the problem can be simplified by adding the following assumptions to those already listed in section 2.1:

- The expansion velocity of the wind is much higher than the sound speed of the gas which is in the range $10-30 \mathrm{~km} \mathrm{~s}^{-1}$. This condition is generally well verified because the observed expansion velocities are not less than $100 \mathrm{~km} \mathrm{~s}^{-1}$.

- The line opacity is much larger than that of the continuum, so that the two transfer problems can be decoupled: this is certainly true in the IR but is not verified at radio wavelengths.

With these assumptions the line radiative transfer is reduced to a local problem in that a photon can interact only with matter at the place of "perfect resonance" where due to systematic motions an atom is perfectly "tuned" for absorbing "that" photon. This is the so-called Sobolev approximation from the name of the russian astronomer who introduced it (Sobolev 1957). Following Castor (1970) the line optical depth can be expressed as

$$
\tau(\nu, \xi)=\frac{\pi e^{2}}{m c} g f \frac{\frac{N_{l}}{g_{l}}-\frac{N_{u}}{g_{u}}}{\nu_{o} \frac{v(\rho)}{c}} R \frac{1}{1+\frac{s^{2}}{\rho^{2}}(\gamma-1)}
$$

where $N_{l}, N_{u}$ and $g_{l}, g_{u}$ are the population and the statistical weight of the lower and upper levels of the transitions. The quantity $\rho$ is the radial distance in the plane $(s, \xi)$, i.e., $\rho^{2}=s^{2}+\xi^{2}$ and like $s$ and $\xi$ is expressed in units of stellar radii. In the case of hydrogen lines, which is the case we will be dealing with, the population of the excited levels is proportional to the square of the ionized matter density and, therefore, the optical depth can be written as

$$
\tau(\nu, \xi)=\tau_{o} \frac{\rho^{-3(\gamma+1)}}{1+\frac{s^{2}}{\rho^{2}}(\gamma-1)}
$$

where

$$
\tau_{o}=\frac{\pi e^{2}}{m c} g f \frac{\frac{N_{l}}{g_{l}}-\frac{N_{u}}{g_{u}}}{\nu_{o} v_{o} / c} R \propto \frac{\dot{M}^{2}}{R^{3} v_{o}^{2}}
$$




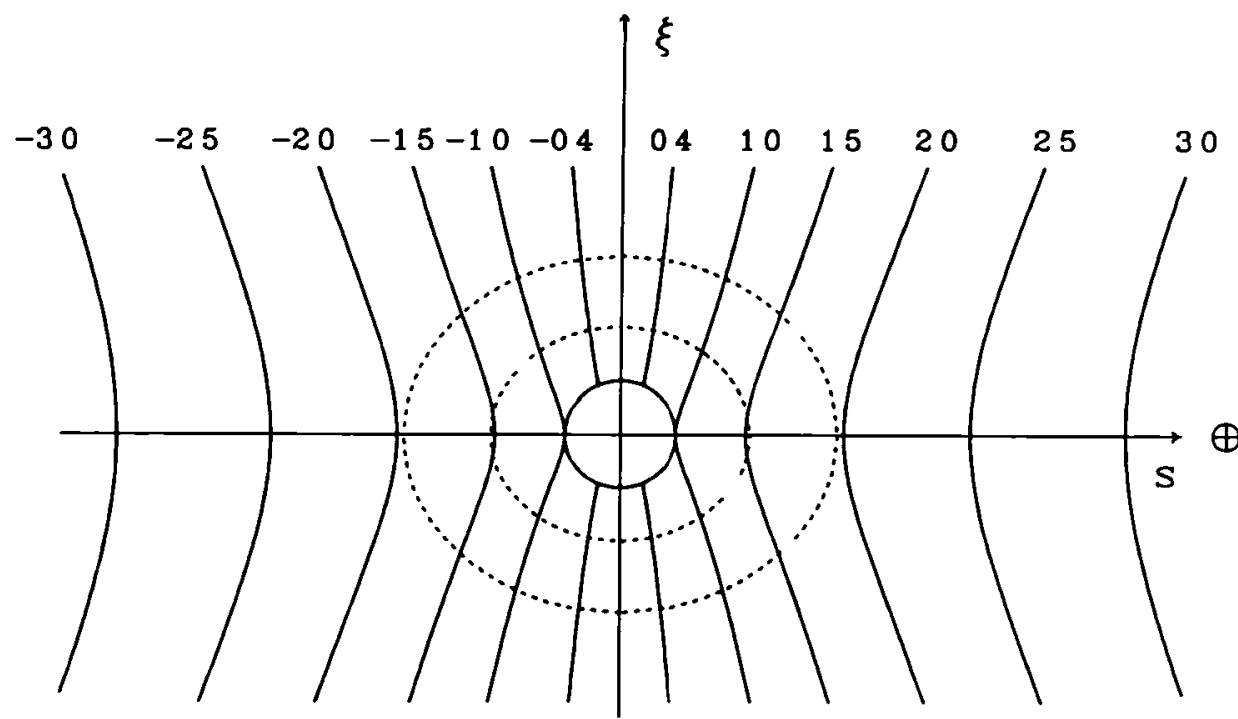

Fig. 3 - The projected surfaces of equal velocity (in units of the initial velocity) for the case of $v \propto r^{1 / 2}$. The loci of constant optical depth are also shown (dashed lines) for $\tau$ values of 10 (inner curve) and 1 (outer curve).

represents the optical depth at $\xi=1$ and at the center of the line, $\nu=\nu_{o}$. Note that all dependences on the frequency are "hidden" in the relationship between the projected velocity, hence the observed frequency, and the geometric coordinates $s$ and $\rho$. As an illustration, let us consider the case of $v \propto r^{1 / 2}$. Figure 3 shows the surfaces of constant projected velocity (as seen by an observer located at "infinite distance" on the right hand side of the figure) as well as some surfaces of constant optical depth. Note that at any value of $\rho$ the optical depth (cf. equation 34) can vary only between $\tau_{o}$ (for $\xi=\rho$ and $s=0$ ) and $\tau_{o} / \gamma$ (for $\xi=0$ and $s=\rho$ ) and, therefore, the surfaces of constant optical depth are almost spherical.

As we have done for the continuum we can distinguish two extreme cases, i.e. optically thin and optically thick cases. However, since the transfer problem is rather more complicated than it is for the continuum, here we will limit ourselves to discuss the simplest concepts and to present the most straightforward results.

In the optically thin case the total (i.e. integrated over the line profile) the line intensity is proportional to the volume emission measure of the wind (and, therefore, to the line optical depth)

$$
I_{t o t} \propto \int n_{e} n_{\imath} 4 \pi r^{2} d r \propto \frac{\dot{M}^{2}}{v_{o}^{2} R}
$$

and the line width is proportional to the initial velocity through a constant which depends exclusively on the index $\gamma$. As long the terminal velocity is much higher than the initial velocity, the half-power-full-width (HPFW) of a line can be expressed as (Panagia et al. 1987):

$$
\mathrm{HPFW}=2^{\frac{4 \gamma+1}{3 \gamma+1}}\left(v_{o} / c\right) \nu_{o}
$$

In the optically thick case there is no simple general solution. Two cases have been considered by Simon et al. (1983), i.e., $\gamma=0$ and $\gamma=1$, who solved the line radiative transfer problem numerically and expressed the results in formulae whose functional dependence 
was derived from simplified theory and the coefficient was determined from a fit to the numerical results. Krolik and Smith (1981) have approached this problem in a general but very simplified manner which we follow here to illustrate the main characteristics of the emission lines formed in an optically thick wind. In such an approach, the size of emitting region is taken to be equal to the radius at which the optical depth becomes unity, i.e.

$$
\xi_{\text {eff }} \simeq \rho(\tau=1, s=0) \simeq \tau_{o}^{\frac{1}{3(\gamma+1)}}
$$

Thus, the intensity at the peak of the line will essentially be that of the black body with such a size, i.e.

$$
I_{\text {peak }} \simeq 4 \pi R^{2} \pi B\left(\nu_{o}\right) \xi_{\text {eff }}^{2} \simeq 4 \pi R^{2} \pi B\left(\nu_{o}\right) \tau_{o}^{\frac{2}{3(\gamma+1)}}
$$

Similarly, the effective width of the line will correspond to the maximum velocity at which the optical depth is still equal to 1 . This occurs at $\xi=0$ (cf. eq. (34)) at a value of $\rho=\rho(\tau=1, \xi=0)$, so that the frequency displacement at which the line is still strong will be

$$
\Delta \nu=\frac{v_{\max }}{c} \nu_{0}=\frac{v_{0}}{c}\left(\frac{\tau_{0}}{\gamma}\right)^{\frac{\gamma}{s(\gamma+1)}} \nu_{0}
$$

This formula essentially provides an estimate of the HPFW, whose functional dependence on the stellar and the wind parameters is

$$
\mathrm{HPFW} \simeq 2 \Delta \nu \propto \dot{M}^{\frac{2 \gamma}{3(1+\gamma)}} R^{-\frac{\gamma}{1+\gamma}} \nu_{o}^{\frac{1}{1+\gamma}}
$$

Hence, the total line intensity can approximately be estimated as

$$
I_{\text {tot }} \simeq I_{\text {peak }} \times \mathrm{HPFW}=4 \pi R^{2} \pi B\left(\nu_{0}\right) \tau_{0}^{\frac{2+\gamma}{3(\gamma+1)}} \nu_{o} \frac{v_{0}}{c}
$$

It is interesting to note that this formula recovers the functional dependences derived by Simon et al. (1983) and extends their validity to any value of $\gamma$. However, due to the crudeness of the approach these formulae are accurate only to within a factor of two as compared with numerical results. More detailed calculations have been made recently by Panagia, Oliva and Felli (1987) both using refined analytical approximations and performing numerical calculations for a large variety of cases. Figures 4 and 5 present the results of the numerical calculations of the total intensity and the HPFW for emission lines formed in a wind where the optical depth is proportional to the square of the density, as appropriate for hydrogen lines. The analytical formulae they derive for the cases of high optical depths agree with the numerical results to better than $30 \%$ in general and better than $10 \%$ in most cases:

$$
\begin{aligned}
I_{\text {peak }} & =4 \pi R^{2} \pi B\left(\nu_{o}\right) \Gamma\left(1-\frac{2}{3(\gamma+1)}\right) \tau_{o}^{\frac{2}{3(\gamma+1)}} \\
I_{t o t} & =4 \pi R^{2} \pi B\left(\nu_{o}\right) \frac{4}{2+\gamma}\left[\Gamma\left(1-\frac{2}{3(\gamma+1)}\right)\right]^{1+\frac{\gamma}{2}} \tau_{o}^{\frac{2+\gamma}{3(\gamma+1)}} \frac{v_{o}}{c} \nu_{o}
\end{aligned}
$$

It also should be noted that at high optical depths, the higher the value of $\gamma$ the wider the lines become both in the HPFW and the extension of wings: this is a natural consequence of the faster increase of the velocity with radius for higher values of $\gamma$. 


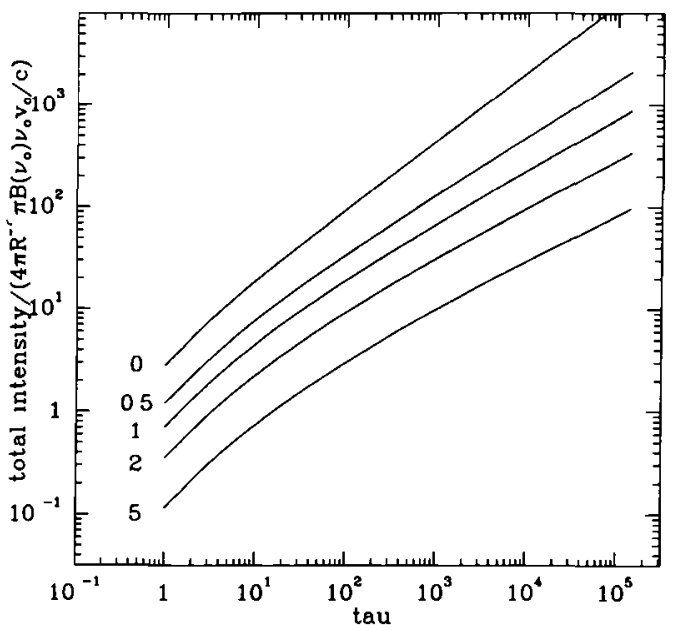

Fig. 4 - The total intensity of emission lines for several values of $\gamma$.

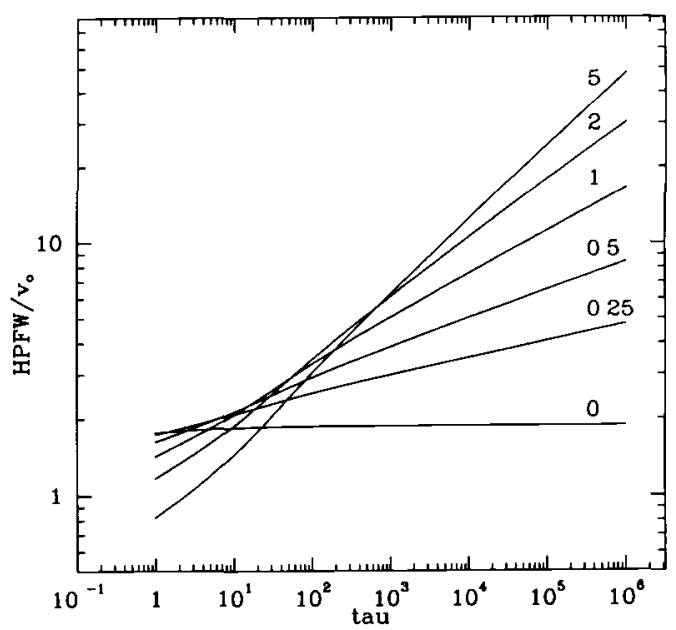

Fig. 5 - The half-power-full-width of emission lines for several values of $\gamma$.

\subsection{Extension to more general cases}

In this section we will briefly consider the effects of releasing some of the basic assumptions we have made in sections 2.1 and 2.3.

\subsubsection{Incomplete ionization}

We can identify two main possibilities:

i) The wind is ionized bounded, i.e. the wind is fully ionized up to a certain radius and becomes mostly neutral beyond that: this the the analog to a Strömgren sphere for the case of an outflow. This problem has been studied in detail by Felli and Panagia (1981) and Simon et al. (1983) who have shown that if such a situation occurs at all, the boundary is likely to be very close to the base of the flow. As a consequence, the IR emission may not be strongly affected while the radio flux can drastically be reduced by orders of magnitude. The maximum rate of mass loss for which a wind can be fully ionized by a star with a Lyman continuum photon flux of $N_{L}$ is (Felli and Panagia, 1981)

$$
\begin{aligned}
\dot{M}_{c}= & 5.75 \times 10^{-6}(1+2 \gamma)^{1 / 2}\left[\frac{N_{L}}{10^{49} \mathrm{~s}^{-1}}\right]^{1 / 2}\left[\frac{v_{o}}{100 \mathrm{~km} \mathrm{~s}^{-1}}\right] \\
& {\left[\frac{R}{10 R_{\odot}}\right]^{1 / 2}\left[\frac{T}{10^{4} K}\right]^{04} \frac{M_{\odot}}{\mathrm{yr}} }
\end{aligned}
$$

Correspondingly the maximum radio flux density which a wind ionized by such a star can emit is

$$
\begin{aligned}
F_{\text {max }}(\text { wind })= & 52.7(1+2 \gamma)^{2 / 3}\left[\frac{\nu}{10 \mathrm{GHz}}\right]^{06}\left[\frac{N_{L}}{10^{49} \mathrm{~s}^{-1}}\right]^{2 / 3}\left[\frac{R}{10 R_{\odot}}\right]^{2 / 3} \\
& {\left[\frac{v_{\infty}}{v_{0}}\right]^{-4 / 3}\left[\frac{d}{1 \mathrm{kpc}}\right]^{-2} \mathrm{mJy} }
\end{aligned}
$$

It is clear then that a wind can never be a very strong source by emitting thermal radiation, i.e. its flux density is unlikely to exceed a few tens of mJy. 
ii) The gas is partially ionized everywhere throughout the wind. Natta et al. (1987) have shown that this situation may occur in the case of low luminosity protostars or young stars. In this case one can still apply the same kind of theory which has been outlined in the previous sections but now one should allow for the incomplete ionization in writing the dependence of the electron and ion density on the radius. In other words, one has to include the extra dependence on the ionization fraction in addition to those already present in the continuity equation. In the simplest case of a constant ionization fraction, all the equations discussed so far still hold with the only change that the mass loss rate should be replaced by the the product of the mass loss rate times the ionization fraction itself. The net effect is that in order to account for any given observed wind emission the required mass loss rate will be $1 /$ (ionization fraction) times higher than in the case of complete ionization.

\subsubsection{The outflow is not spherically symmetric:}

This case has been considered in quite some detail by Schmid-Burgk (1982) and Reynolds (1986). As for the slope of the continuum, the lack of spherical symmetry may affect it appreciably only if the fraction of solid angle filled by the flow varies systematically with the radius. For example, Reynolds has shown that in the case of collimated jets with cross section varying like $w(r)=w_{o}\left(r / r_{o}\right)^{\eta}$ and a density within the jet $n_{e}(r)=n_{o}\left(r / r_{o}\right)^{-\beta}$ the radio continuum spectral index is given by

$$
\alpha(\beta, \eta)=2-2.1 \frac{1+\eta}{2 \beta-\eta}
$$

As for the absolute value of the flux it is found that, irrespective of the details of the source geometry, the formulae derived for a spherically symmetric wind are still valid, to within a factor of two, for more complex configurations as long as any ratio of the structural length scales in the source does not exceeds a value of about 10 . In other words, only highly anisotropic structures can give results appreciably deviant from the predictions of the spherically symmetric models. Among these alarming cases one can consider a wind which consists solely of a number of narrow jets: in this case the radiating efficiency can be higher than in a spherical wind by as much as an order of magnitude and, therefore, the mass loss rate derived with spherical wind formulae may be strongly overestimated. For example, Reynolds (1986) has shown that a narrow conical jet with opening angle $\theta_{0}$ and seen with an inclination $i$ can produce the same radio flux with a much smaller mass loss rate given by

$$
\dot{M}(j e t)=\dot{M}(\text { spherical wind }) \times 0.20 \theta_{o}^{3 / 4}(\sin i)^{-1 / 4}
$$

\subsubsection{Time variability}

One of the basic assumptions is that the mass loss process is steady with time. On the other, variability is an observed fact and the more so in the case of young stellar objects. A discussion of the main effects has recently been presented by Felli et al. (1985b). Summarizing it briefly here, one can distinguish two cases: i) variation of the mass loss rate, and ii) variation of the ionization. In the former case one expects to detect short term variability in the optical and IR but not in the radio because even if the $\dot{M}$ variation is instantaneous the transit time of the front is

$$
t_{\text {transit }} \simeq R_{\text {eff }} / v=19.3\left[\frac{R_{\text {eff }}}{10 R_{\odot}}\right]\left[\frac{v}{100 \mathrm{~km} \mathrm{~s}^{-1}}\right]^{-1} \text { hours }
$$


and, therefore, it is of the order of a few hours in the near IR but it can be of the order of weeks or months in the radio. Whether or not one sees any variation at all in the radio depends on the time scale of the intrinsic variation: if it occurs over a time much shorter than a few weeks, then the variations will completely be averaged out in the radio. In the case in which the ionization varies, the situation is different. Again assuming that the ionizing agent undergoes a sudden variation, the time scale of the phenomena is set by the recombination time which varies like a higher power of the radius:

$$
\begin{aligned}
t_{\text {rec }}= & \frac{1}{n_{e} \beta_{2}}=7.4\left[\frac{\dot{M}}{10^{-6} M_{\odot} \mathrm{yr}^{-1}}\right]^{-1}\left[\frac{R_{e f f}}{10 R_{\odot}}\right]^{2} \\
& {\left[\frac{v}{100 \mathrm{~km} \mathrm{~s}^{-1}}\right]\left[\frac{\mu_{e}}{1.2}\right]\left[\frac{T}{10^{4} K}\right]^{0.8} \text { seconds } }
\end{aligned}
$$

Therefore, one expects a much more prompt reaction in the optical and IR, which originate in the inner and denser layers, than in the radio. These points stress the importance of making simultaneous measurements at widely different frequencies in order to understand the nature and the origin of the variability.

\subsubsection{Accretion}

All throughout this theoretical section we have assumed that the emitting gas is flowing away from a young star. But the reverse situation, i.e. mass infall or accretion, may occur since we are dealing with just forming objects. And its occurrence is actually detected in some instances by the observation of inverse $\mathrm{P}$ Cyg profiles of optical lines, i.e. lines with a red absorption and a blue emission, which are the unambiguous sign of gas falling onto the stellar surface. In these case, most of the theory remains valid apart for an obvious change in the velocity sign. In fact, one cannot distinguish the velocity sign, whether the mass flows in or out of a star, from observations in the continuum because its flux is solely determined by the density radial behaviour which is invariant with respect to a sign inversion as long as the modulus variation of the velocity stays the same. On the other hand, in the case of accretion one does not expect a lower velocity closer to the star because gravity will unavoidably accelerate the matter as it falls onto the star. As a consequence, the expected velocity slope is negative $(\gamma<0)$ whereas in the case of a wind both the theory and the observations suggest an outwardly acceleration, i.e. $\gamma>0$. Of special interest is the case of accretion in free fall conditions which entails $v \propto r^{1 / 2}$. It is easy to verify that with the resulting density distribution $n \propto r^{-3 / 2}$ the volume emission measure

$$
\int n_{e} n_{z} d V \propto n_{o}^{2} r_{o}^{3} \ln \left(r / r_{o}\right)
$$

would diverge with increasing radius. Therefore, either the distance at which the inflow originates is suitably small or the gas will unavoidably be ionization bounded, i.e. neutral in the outer parts of the flow, for any accretion rate. For this reason the continuous spectrum will remain essentially flat even at those frequencies at which the flow is optically thick until the effective emitting radius will become comparable to the outer boundary. Felli et al. (1982) give an approximate expression for the radio emission. After some manipulation it can be written as

$$
\begin{aligned}
F_{\nu} \text { (accretion) }= & 8.18\left[\frac{\nu}{10 \mathrm{GHz}}\right]^{-01}\left[\frac{T}{10^{4} K}\right]^{-035} \\
& {\left[\frac{\dot{M}_{a c c r}}{10^{-7} M_{\odot} \mathrm{yr}^{-1}}\right]\left[\frac{M}{M_{\odot}}\right]^{-1}\left[\frac{d}{1 \mathrm{kpc}}\right]^{-2} \ln \left(\frac{r_{\infty}}{r_{c}}\right) \mathrm{mJy} }
\end{aligned}
$$


where $\dot{M}_{\text {accr }}$ is the accretion rate, $M$ is the stellar mass, $r_{c}$ is the radius at which the radial optical depth is equal to $3 / 4$ :

$$
\begin{aligned}
r_{c}= & 3.66 \times 10^{14}\left[\frac{\nu}{10 \mathrm{GHz}}\right]^{-105}\left[\frac{T}{10^{4} K}\right]^{-068} \\
& {\left[\frac{\dot{M}_{a c c r}}{10^{-7} M_{\odot} \mathrm{yr}^{-1}}\right]\left[\frac{M}{M_{\odot}}\right]^{-1}\left[\frac{\mu_{e}}{1.2}\right]^{-1} \mathrm{~cm} }
\end{aligned}
$$

$r_{\infty}$ is the outer boundary, generally corresponding to the accretion radius

$$
r_{\infty} \simeq\left(\frac{v_{\text {escape }}}{v_{\text {turb }}}\right)^{2} r_{o}=2.65 \times 10^{16}\left[\frac{M}{M_{\odot}}\right]\left[\frac{v_{t u r b}}{1 \mathrm{~km} \mathrm{~s}^{-1}}\right] \mathrm{cm}
$$

$v_{t u r b}$ being the turbulent velocity of the cloud where the accretion originates. The spectrum described by equation (51) is essentially flat, corresponding to spectral indexes between -0.1 to +0.1 . This is possible because, although the central parts can be quite optically thick, still a considerable fraction of the flow remains optically thin. Equation (51) is valid as long as $r_{c} \ll r_{\infty}$ or, conversely, as long the frequency is higher than

$$
\begin{aligned}
\nu_{c}= & 169\left[\frac{T}{10^{4}}\right]^{-064}\left[\frac{\dot{M}_{a c c r}}{10^{-7} M_{\odot} \mathrm{yr}^{-1}}\right]^{095}\left[\frac{M}{M_{\odot}}\right]^{-143} \\
& {\left[\frac{v_{t u r b}}{1 \mathrm{~km} \mathrm{~s}^{-1}}\right]^{190}\left[\frac{\mu_{e}}{1.2}\right]^{-095} \mathrm{MHz} }
\end{aligned}
$$

At lower frequencies the spectrum will tend to the ordinary Rayleigh-Jeans tail of a black body, i.e. $F_{\nu} \propto \nu^{2}$. It is clear then that the spectrum will remain essentially flat down to frequencies well below a gigahertz and then decline like $\nu^{2}$ at lower frequencies. Therefore, judging from the radio spectrum any accreting star could be mistaken for a compact HII region, except that the widths of the emission lines would be drastically different, i.e. several hundreds of $\mathrm{km} \mathrm{s}^{-1}$ for an accretion flow as compared with the few tens of $\mathrm{km} \mathrm{s}^{-1}$ expected for an HII region.

\subsection{Non-thermal emission}

The main non-thermal process which is relevant for young stars is synchrotron radiation. It is produced by electrons spiraling in a magnetic field and is regarded as a non-thermal process because it is an efficient process only with energetic electrons and these are abundant enough in non-thermal situations. This aspect makes much harder to uniquely define the parameters of a synchrotron emitting source or even to define the most probable situations, essentially because there are too many free parameters and too many unknowns. Therefore, here we will not discuss this emitting mechanism in detail but rather we shall limit ourselves to describing its main properties. More detailed summaries and discussions on the non-thermal emission processes in stars can be found in Dulk (1985), White (1985) and André (1987). The main characteristics of synchrotron radiation are i) a typical power law spectrum which, unlike to thermal spectra, increases with wavelength (until it starts becoming self-absorbed) ii) high polarization and iii) high brightness. This last property is due to the fact that very high energy particles are involved and, therefore, the limiting brightness temperature can be accordingly very high. Because of the same reason, a non-thermally emitting region can be much more compact than a thermal one. 
Both the small size, which implies a short transit time, and the high energy density, which implies a short energy loss time, concur in making the typical time scale of temporal variations of the non-thermal radio emission quite short. With the parameters appropriate for a star, such timescales range from hours to few days: this is orders of magnitude faster than it is possible in a thermal region. Therefore, short term variability can be taken as a fourth distinctive property of non-thermal emitting sources.

\section{RADIO AND INFRARED OBSERVATIONS OF YOUNG STARS}

\subsection{The "well behaved" case of S 106-IRS4}

One of the best studied young stars is S 106-IRS4. As its name suggests, it is a star associated with the HII region S 106 and has been discovered in the IR as a point like source (Sibille et al. 1975; Allen and Penston, 1975). In fact it cannot be seen in the optical because of about 21 magnitudes of visual extinction in front of it. The whole region has been the subject of a number of studies in the IR and in the radio (considering only papers relevant to the present discussion, see e.g.: Simon and Fischer 1982, Bally et al. 1983, Felli et al. 1984, 1985c and references therein). The radio observations show $S$ 106-IRS4 to be a point like source (Bally et al. 1983; cf Fig. 6) still unresolved at $22 \mathrm{GHz}$ (diameter $\leq 0.15^{\prime \prime}$, Felli et al. 1985c) and with a spectral index $0.7 \pm 0.1$ (cf Fig. 7 ). In the infrared, shortward of about $10 \mu \mathrm{m}$, it displays a clear excess relative to the stellar emission (Felli et al. 1984) and hydrogen emission lines (Bro, and Bry; Simon and Fischer 1982, Felli et al. $1985 \mathrm{c}$ ) whose HPFW is around $150 \mathrm{~km} \mathrm{~s}^{-1}$ and whose intensity ratios differ markedly from those of lines formed in HII regions. All of these aspects fit perfectly the pictupe of a star undergoing conspicuous mass loss. Combining all the available information Fell 1 et al. (1984) deduced the following parameters for the star and the wind, for an assumed distance of $600 \mathrm{pc}$ :

$\begin{array}{lll}\text { Star } & \text { Effective Temperature, } T_{\text {eff }} & 28000 \mathrm{~K} \\ & \text { Radius, R } & 12 R_{\odot} \\ \text { Luminosity, L } & >2 \times 10^{4} L_{\odot}\left(\sim 10^{5} L_{\odot}\right) \\ & \text { Lyman Continuum Photon Flux, } N_{L} & 1.7 \times 10^{48} \text { photons s }^{-1} \\ \text { Inferred Stellar Mass } & >15 M_{\odot} \\ & \text { Matching Spectral Type } & 09.5 \mathrm{~V}-\mathrm{B} 0 \mathrm{III} \\ & & \\ \text { Wind } & \text { Mass Loss Rate, } \dot{M} & 2.6 \times 10^{-6} M_{\odot} \mathrm{yr}^{-1} \\ & \text { Velocity Index, } \gamma & 0-0.25 \\ & \text { Initial Velocity, } v_{o} & 40-80 \mathrm{~km} \mathrm{~s}^{-1} \\ & \text { Terminal Velocity, } v_{\infty} & \sim 300 \mathrm{~km} \mathrm{~s}^{-1}\end{array}$

Thus, S 106-IRS4 looks like the perfect "theorist's dream", i.e. the object whose observations fit all theoretical predictions. We shall see in the following sections that reality is not always so nice nor so simple. Even in this case the results are not "boring": what at first glance seems just a replica of an ordinary early type star, after a deeper exam reveals a number of peculiar properties:

i) The ratio $\dot{M} / L$ is higher than $3 \times 10^{-11} M_{\odot} L_{\odot}^{-1} \mathrm{yr}^{-1}$ : This is already an order of magnitude higher than it is measured in "normal" main sequence (MS) and supergiant stars (Tanzi et al. 1981) and is comparable to the values of WR stars $\left([\dot{M} / L]=4 \times 10^{-11} M_{\odot} L_{\odot}^{-1}\right.$ 


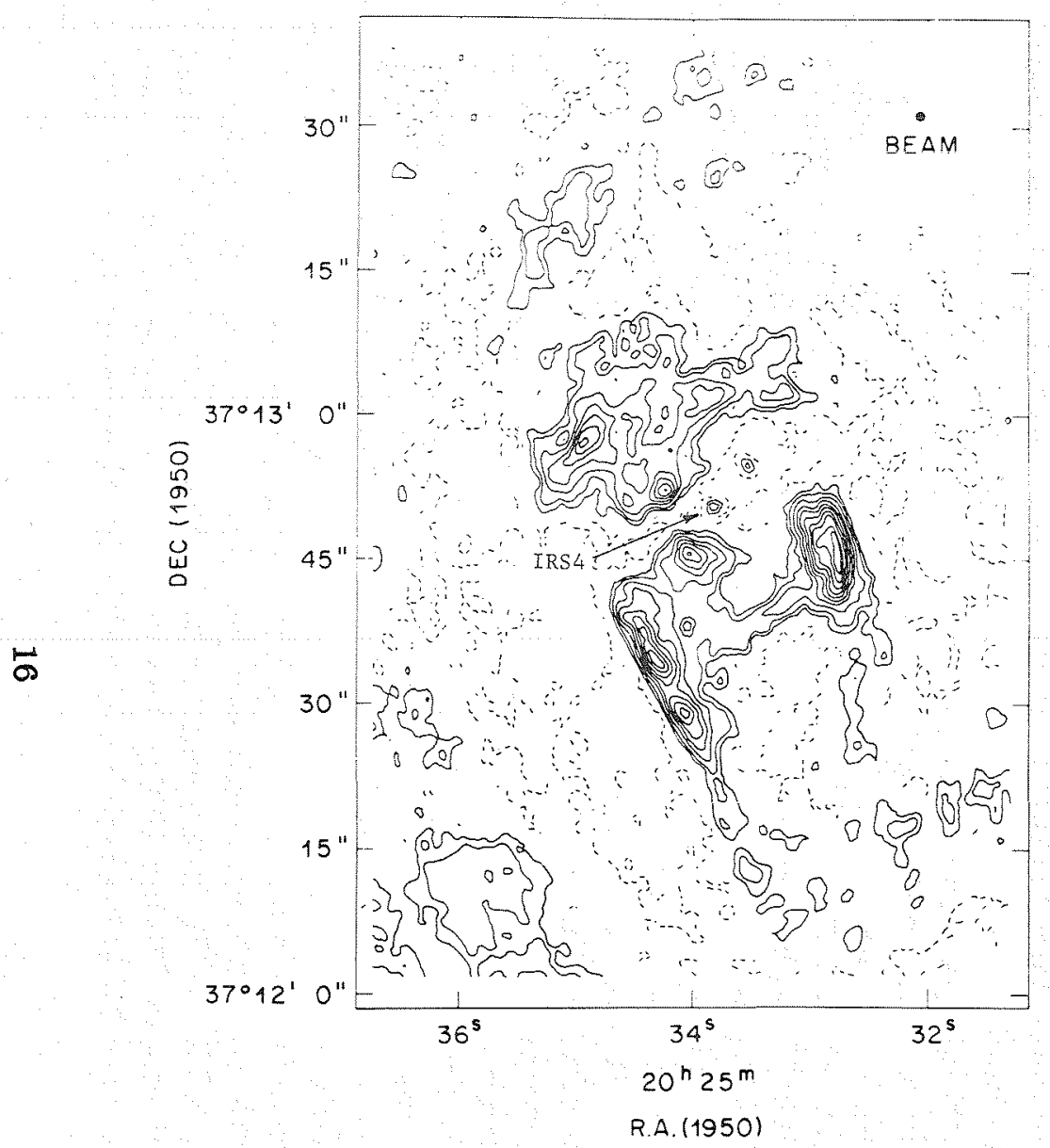

Fig. 6 - The VLA radio map of the S 106 region at $15 \mathrm{GHz}$ (Bally et al. 1983).

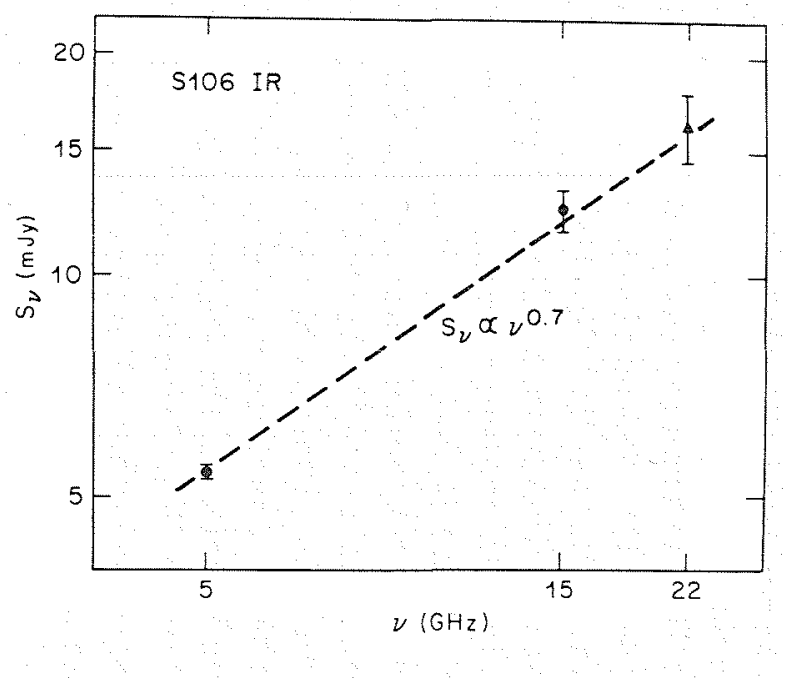

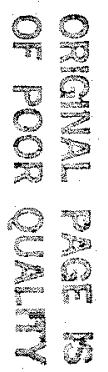

Fig. 7 - The radio spectrum of S 106-IRS4 (from Bally et al. 1983). 
$\mathrm{yr}^{-1}$; Barlow et al. 1981). ii) The momentum carried by the wind exceeds that available in the radiation field: $\left(\dot{M} v_{\infty}\right) /(L / c) \sim 2$. This is intermediate between ordinary OB stars, that have ratios lower than 0.5 , and WR stars for which that ratio is higher than 10 . iii) The terminal velocity, $v_{\infty}=300 \mathrm{~km} \mathrm{~s}^{-1}$, is much lower than that measured in MS and SG stars of comparable luminosity $\left(v_{\infty} \simeq 1500 \mathrm{~km} \mathrm{~s}^{-1}\right.$, see e.g. Panagia and Macchetto, 1982) These aspects suggest that S 106-IRS4 represents an especially "active", short-lived, premain-sequence phase of the evolution of a moderately massive star. We will see in section 4 that most PMS stars seem to pass through such a phase before reaching the MS. Another interesting aspect is the marked absence of radio emission in a strip about $5^{\prime \prime}$ wide passing through the star in the SE-NW direction (cf Fig. 6). It can only be due to lack of ionizing photons in that direction because identical gaps are seen also in the IR, thus excluding the hypothesis of dust absorption occurring in a small disk/ring around the star. The most "natural" explanation is that of a genuine anisotropy of the stellar UV radiation, which is minimum on the equatorial plane and maximum toward the poles (Felli et al 1984). The orientation of this "void" region is perpendicular to the direction of the large scale, bipolar flow and is essentially coplanar with the disk-like molecular region which projects in front of the star. Therefore, we see that one and the same geometric symmetry exists from a very small scale, i.e. the stellar radius $\sim 10^{12} \mathrm{~cm}$, up to a very large scale, i.e. the size of the molecular disk of about $3 \times 10^{18} \mathrm{~cm}$. The high degree of "organization" of very different scales suggests that all of these aspects are intimately connected with each other and have a common origin.

\subsection{General results of radio observations}

Essentially all possibilities considered theoretically in the previous sections are observed in different objects. André (1987) in his review of the radio emission from young stars has defined four broad classes which are based on observed characteristics and can place the various objects into physically meaningful categories:

1) Steady sources, unresolved, steep spectrum with positive spectral index $(\alpha>0.5)$ : thermal wind. Examples of this class are, in addition to the obvious S 106-IRS4, the BN object (Moran et al. 1983), Lk $\mathrm{H} \alpha 101$ (Brown et al. 1976) Lk $\mathrm{H} \alpha 234$ (Wilking et al 1986), GL 490 (Campbell et al. 1986), CRL 961 (Snell and Bally 1986) and component \#2 of Cep A (Hughes, 1985). All of these sources can be explained in terms of spherically symmetric, fully ionized winds with either constant or slightly increasing velocity.

2) Steady source, resolved, flat spectrum: thermal jet or ultracompact HII region. Examples: G129.58-0.04, NGC 2071, S 68-FIRS1, L1551-IRS5 (Snell and Bally 1986). The radio spectrum of this type of sources does not follow a simple power law, the spectral index being steeper at lower frequencies. An alternative to the HII region model is that such a spectrum is due to accretion.

3) Steady source, unresolved, flat spectrum: thermal accretion flow or non-thermal emission? Typical examples are S1, VS 14 and WL 5 in the $\zeta$ Oph cloud (André et al. 1987). The stable emission observed for these objects cannot be readily explained as due to flare activity but, on the other hand, being unresolved with the VLA their surface brightness seems to be too high for a ordinary thermal source.

4) Variable source, unresolved: non-thermal stellar flares. Examples are: DoAr 21 (= ROX 8), ROX 31 (Feigelson and Montmerle 1985); V410 Tau, HP Tau/G2 and HP Tau/G3 (Cohen and Bieging 1986). The strong variability on short time scales identifies these sources as definitely non-thermal. André, Montmerle and Feigelson (1987) note that 
their fluxes are comparable to those of post-main sequence RS CVn systems and suggest that they may be associated with the most luminous $\mathrm{X}$-ray selected pre-main-sequence stars.

As remarked by Andre (1987) the objects in classes 1) and 2), i.e. those which do have stellar winds, show some definite evidence of large scale outflows, e.g. optical jets and/or association with $\mathrm{HH}$ objects for T Tau stars, bipolar flows for more powerful IR stars, so that they can easily fit with the same picture as drawn for S 106-IRS4 (cf. section 3.1).

\subsection{Radio Surveys}

If now we have an idea of what individual stars may do, still it isn't clear how many of them do that and for how long, i.e. we know about the phenomena that may occur but we cannot place them in the evolutionary history of a star. A step forward in that direction can be taken by making complete surveys in regions of star formation. In such a way, not only can one determine the properties of individual sources which were detected but also consider the "non-detections" to perform statistics which can tell us about the duration of the various phases in different classes of objects. Among the studies of this sort done in recent years I have selected three that are particularly relevant for our interests.

1) Breging, Cohen and Schwartz (1984) - They made VLA observations at 4 frequencies $(1.5,4.9,15$ and $22.5 \mathrm{GHz})$ of all $\mathrm{T}$ Tau stars with $\log L / L_{\odot}>0.2$ associated with the Taurus-Auriga dark cloud ( 46 objects). Their results can be summarized as follows:

- 12 stars out of the $46(26 \%)$ were detected at one frequency at least.

- For 8 of them the spectral index was determined:

3 had a steep spectrum $(\alpha>1)$ which could be interpreted as thermal emission; however, for one of them, V410 Tau, the fast variability indicates a non-thermal origin of the emission.

2 had a spectrum consistent with a stellar wind $(\alpha \sim 0.6)$

2 had a flat spectrum but were unresolved: they could be explained as either nonthermal or accretion emission.

1 had a negative spectral index (HP Tau/G2, $\alpha=-0.35$ ) which speaks clearly of non-thermal radiation.

Therefore, in about half of the cases the emission is of thermal origin and, among these, in about half it originates from stellar winds.

- The missed detection of 34 of the surveyed stars implies an upper limit to their mass loss rate of $\dot{M} \sim 2 \times 10^{-8} M_{\odot} \mathrm{yr}^{-1}\left[\mathrm{v} / 200 \mathrm{~km} \mathrm{~s}^{-1}\right]$.

- All of the 5 stars associated with $\mathrm{HH}$ objects do emit in the radio.

2) Snell and Bally (1986) - They surveyed (complete VLA mapping at 1.4, 5 and $15 \mathrm{GHz}$, to limiting fluxes of about $0.3 \mathrm{mJy}$ at $5 \mathrm{GHz}$ ) 12 regions containing molecular outflows. The main results are:

- Sources were detected in 8 out of the 12 surveyed regions.

- A total of 43 sources were detected. However, based on a statistical argument of source counts, it is concluded that about half of them are likely to be extragalactic.

- 15 of the detected sources were identified with IR sources. In particular, all IR sources associated with the outflows having a bolometric luminosity higher than $100 L_{\odot}$ were detected (9 objects). In total, the IR sources detected in the radio amount to about $2 / 3$ of all genuine sources contained in these outflow regions.

- For 7 sources the spectral index was determined: 
3 had a spectral index and a size fully consistent with a stellar wind interpretation: CRL 961, Lk $\mathrm{H} \alpha 234$ in addition to our friend S 106-IRS4.

4 had a flat index: two (L1551 IRS5 and NGC 2071 IRS 1-3) are clearly extended and, therefore, are likely to be compact HII regions, whereas all possibilities, except a stellar wind hypothesis, remain possible for the other two.

- For all sources, if the radio emission is formed by a steady, fully ionized wind, the implied mass loss parameters fail by more than an order of magnitude to explain the energetics of the associated molecular outflows.

3) André, Montmerle and Feigelson (1987) - They made a VLA survey of the $\rho$ Oph dark cloud, covering an area of 4 square degrees at $1.4 \mathrm{GHz}$ (limiting fluxes 1-3 mJy) and observing the central part also at $5 \mathrm{GHz}$ with a limiting flux of $1 \mathrm{mJy}$. Over the whole region, they detected 93 objects of which they judged that only 13 were truly stellar objects. Grouping the detected objects according to a variety of properties, they find:

- 7, i.e. $\sim 60 \%$ of the sources, can be identified with near or far IR sources. However, among the three classes defined by Lada (1986; see section 3.4) only class III objects are detected: in particular, the detection rates are $0 / 5$ for class I, $0 / 21$ for class II and $4 / 6$ for class III. Three more IR sources detected in this survey could not be classified within the Lada's scheme.

- 10 , i.e. $77 \%$ of the detected stellar sources, have a spectral index either flat or increasing with frequency. One of them, DoAr 21 is known to be non-thermal because of its variability. Most of the others are likely to be thermal sources.

- 5 are associated with X-ray stellar sources. Since this cloud contains 47 such stars, only about $10 \%$ of the $\mathrm{X}$-ray stars emit in the radio.

- None of the 26 visible PMS (emission-line or T Tau) stars associated with the cloud was detected. This places an upper limit to their mass loss rate of $\dot{M}<7 \times 10^{-8} M_{\odot}$ $\mathrm{yr}^{-1}\left[\mathrm{v} / 200 \mathrm{~km} \mathrm{~s}^{-1}\right]$.

\subsection{Infrared observations}

Infrared measurements are important for the study of young stars for two main reasons: first because the high extinction of star forming clouds, that prevents us from "penetrating" deeply into them at optical wavelengths, becomes less and less important at longer wavelengths. The second reason is that, especially for very young objects, the original circumstellar cocoon is still so thick that the bulk of the stellar energy is actually radiated in the infrared. In fact, most of the high luminosity PMS stars can only be detected in the IR, because their short evolutionary time makes it hard for the circumstellar material to dissipate before the star has reached the main sequence. A historically important example is the case of the Becklin and Neugebauer object (BN) which was discovered as an extremely cool object $(T \sim 700 \mathrm{~K})$ appearing in a map of the Orion Nebula at $2.2 \mu \mathrm{m}$ and was immediately recognized as a possible protostar (Becklin and Neugebauer, 1967).

A systematic study of the energy distributions of young stellar objects has been done by Wilking and Lada (1983) and Lada and Wilking (1984) who made broad-band photometric observations of the embedded population in the core of the $\rho$ Oph dark cloud. From an analysis of the derived energy distributions $\left(\lambda F_{\lambda}\right.$ vs $\left.\lambda\right)$ they identified three main morphological classes (see fig. 8):

Class I - Sources with energy distributions broader than a black body and which are rising longward of $2 \mu \mathrm{m}$. 

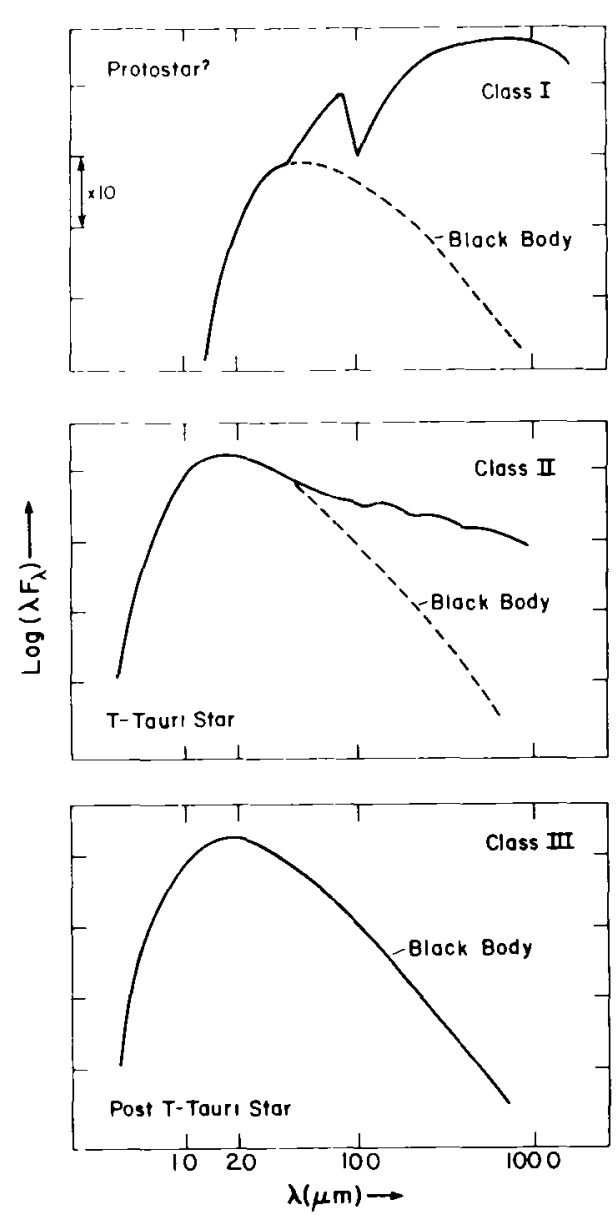

Fig. 8 - Schematic representation of the classification of young stellar objects on the basis of their IR spectra (Lada and Wilking 1984).

Class II - Sources with an energy distribution broader than a black body but which is flat or decreasing longward of $2 \mu \mathrm{m}$.

Class III - Sources whose energy distributions can be modeled with reddened black bodies and show no or little excess near infrared emission.

Class I sources were all invisible and deeply embedded in the cloud whereas class II and III sources were associated mostly with visible stars. In particular, nearly all previously known class II objects were classified as T Tau stars. Moreover, the data available in the literature for a number of stars of low and moderate luminosity $\left(L<10^{3} L_{\odot}\right)$ fit equally well in this classification scheme. In particular, inspecting the Rydgren et al. (1984) catalog of the infrared spectra for $61 \mathrm{~T}$ Tau stars in the Taurus-Auriga complex one finds that among the 41 stars for which the wavelength coverage is complete enough to classify them, 34 falls in class II, 5 in class III and none in class I. Lada (1986) suggests that such an empirical sequence may represent an evolutionary sequence in which class I objects are protostars, just formed and still deeply embedded in their original cloud. The circumstellar envelopes dissipate gradually with time, thus producing class II and III morphologies. The fact that many (but not all) class I objects and many (but not all) $\mathrm{T}$ Tau stars are associated with energetic outflows and/or stellar wind activity suggests that these processes represent the agents that dissipate the circumstellar envelopes and drive the evolution from class I to class III. From all of this it is clear that, due to the strong dust emission, in most cases (class I and class II objects) one cannot use IR continuum measurements to safely estimate the properties of the stellar wind. On the other hand, line emission is not affected by the 
presence of dust, except for the obvious effect of extinction. In addition, lines have the great advantage of carrying information about the velocity of the wind; such an information is completely lost in the continuous radiation. Therefore, IR emission lines are very precious for specifying the detailed properties of the stellar winds. The most intense lines observed from PMS stars are generally hydrogen emission lines such as $\operatorname{Br} \alpha(4.05 \mu \mathrm{m}), \operatorname{Br} \gamma(2.17$ $\mu \mathrm{m})$ or $\operatorname{Pf} \gamma(3.74 \mu \mathrm{m})$. By using the theory outlined in section 2.3 one can determine both the mass loss rate and the characteristics of the velocity field in the wind. A number of searches have been carried out on PMS stars of all luminosities from $\sim 1 L_{\odot}$ up to $10^{6} L_{\odot}$ (among the most recent studies we recall Thompson 1982, Simon et al. 1983, Thompson et al. 1983, Persson et al. 1984, Smith et al. 1987, Thompson 1987, Evans et al. 1987, Persson et al. 1987). The main result is that strong lines are indeed observed, with a strength much higher than predicted from the measured radio flux if the emitting region is assumed to be optically thin at all relevant wavelengths (the so-called HII region theory). Such a result, surprising at first glance, is the obvious consequence of the wind properties, in particular the high optical depth. In fact, the wind is optically thick longward of few microns and the optical depth in the radio is several orders of magnitude higher than in the IR. Therefore, while both the IR and the radio fluxes are lower than in the optically thin case, the effect is much more conspicuous in the radio where the optical depth is higher. Hence, the ratio IR/radio in a wind can become considerably higher than it is in an HII region.

Another important result obtained from line measurements is the realization (e.g., Thompson 1982) that the ionization in the wind does requires a large number of ionizing photons, in many cases highly in excess to what the star can supply in the form of Lyman continuum photons (i.e., photons capable of ionizing the ground level of hydrogen, $h \nu>$ $13.6 \mathrm{eV})$. This problem will be discussed in some detail in the next section.

\section{STATISTICAL PROPERTIES OF STELLAR WINDS FROM YOUNG STARS}

Here, we discuss the available information on winds from PMS stars to try to understand their nature and their relation to the process of star formation. A more complete account of this analysis can be found in Panagia (1985).

The data were collected from various sources in the literature, namely Cohen et al. (1982), Felli et al. (1982 and 1984), Persson et al. (1984), Simon et al. (1983), Thompson (1982), Thompson et al. (1983). The derived parameters, such as the mass loss and ionization rates, were directly taken from the original articles and may not be fully consistent with each other in that various authors sometimes have used different models to interpret their data. No attempt was made to 'homogeneize' the data and the possible discrepancies arising from the use of different models have been regarded as additional errors which sum up to the observational uncertainties.

The quantities that we can consider to define the properties of PMS star winds are:

1. The total radiative luminosity, $L_{t o t}$, which includes the energy observed in the visual and, possibly, in the infrared plus the energy needed to ionize the wind (see point 3 ).

2. The maximum expansion velocity, $v_{\max }$, as deduced from line profile observations.

3. The wind ionization luminosity, $L_{z o n}$, i.e. the energy required to keep the wind gas steadily ionized. It is computed as the product of the number of ionizations occurring in 
the wind (as deduced from IR/optical line or IR/radio continuum observations) times the $\mathrm{H}$ ionization energy, $13.6 \mathrm{eV}$.

4. The mass loss rate, $\dot{M}$. It is deduced from either line measurements (mostly IR lines but sometimes optical, e.g. $\mathrm{H} \alpha$ ) or IR/radio continuum data.

5. The momentum flux defined as the product of the mass loss rate times the maximum wind velocity. The actual momentum carried away by the wind can be higher than this estimate, by a factor of $\sim 2$, because part of the momentum is used to win the gravitational force (Abbott, 1980).

6. The 'mechanical' luminosity, $L_{k_{2} n}$, of the outflowing wind defined as the product of the mass loss rate times $1 / 2 v_{\max }^{2}$. Note that the total kinetic energy carried by the wind away from the star may be higher because part of it has to be used to overcome the gravitational potential and part may have been transformed into heat.

The total radiative luminosity will be used as the independent variable because it is the observational quantity which is essentially determined by the stellar mass and, therefore, best characterizes the properties of the star irrespective of the presence of the wind. Plots of the other quantities as a function of $L_{t o t}$ are displayed in Figures 9-13.

The first interesting result is that in all cases the various quantities follow well defined trends as a function of $L_{t o t}$. Clearly, there is some dispersion present in all plots but it is always much smaller than the total variation of the involved quantities and, therefore, there is no doubt that the apparent correlations are real.

The second important aspect is that there is good continuity in the properties of the winds of PMS stars over as much as six orders of magnitude in the luminosity from, say, about $1 L_{\odot}$ up to more than a million solar luminosities and correspondingly for stellar masses in the range from about $1 M_{\odot}$ up to almost $100 M_{\odot}$. Such a continuity of properties over a quite broad interval strongly suggests a great uniformity in the processes which induce and govern stellar winds in the PMS phase.

Looking at individual plots, we see (Fig. 9) that the maximum expansion velocity is of few hundred $\mathrm{km} \mathrm{s}^{-1}$ for all stars. Also, it is apparent that $v_{\max }$ is either lower or, at most, equal to the escape velocity from the stellar surface $\left(v_{e s c}=2 \mathrm{GM} / \mathrm{R}\right)$ : this is true whether we compute $v_{\text {esc }}$ using the stellar parameters of main sequence (MS) stars or if we adopt larger radii for any given luminosity as appropriate for PMS stars (hereafter we assume that a typical PMS star has an effective temperature a factor of 1.58 , or $0.2 \mathrm{dex}$, lower than, and a luminosity equal to, that of a MS star of equal mass). Moreover, the observed velocities are much lower than those measured for the winds of MS stars, at least for those of high luminosity mass (e.g. Panagia and Macchetto, 1982). This suggests that the acceleration mechanism is different from that operating in MS stars. Furthermore, the fact that the terminal velocity is never higher than the escape velocity at the stellar surface suggests that the acceleration process is rather gradual and must operate up to relatively large distances from the stellar surface.

Looking at Figure 10 we see that $L_{20 n}$ is very well correlated with $L_{\text {tot }}$ over almost 6 orders of magnitude with a modest dispersion of $\sim \pm 1 / 2$ dex. The best-fitting power-law relationship is

$$
L_{\text {ton }} \propto L_{\text {tot }}^{12 \pm 02}
$$

Alternatively, the experimental correlation can be expressed as

$$
L_{\text {son }} / L_{\text {tot }} \simeq 8 \%
$$



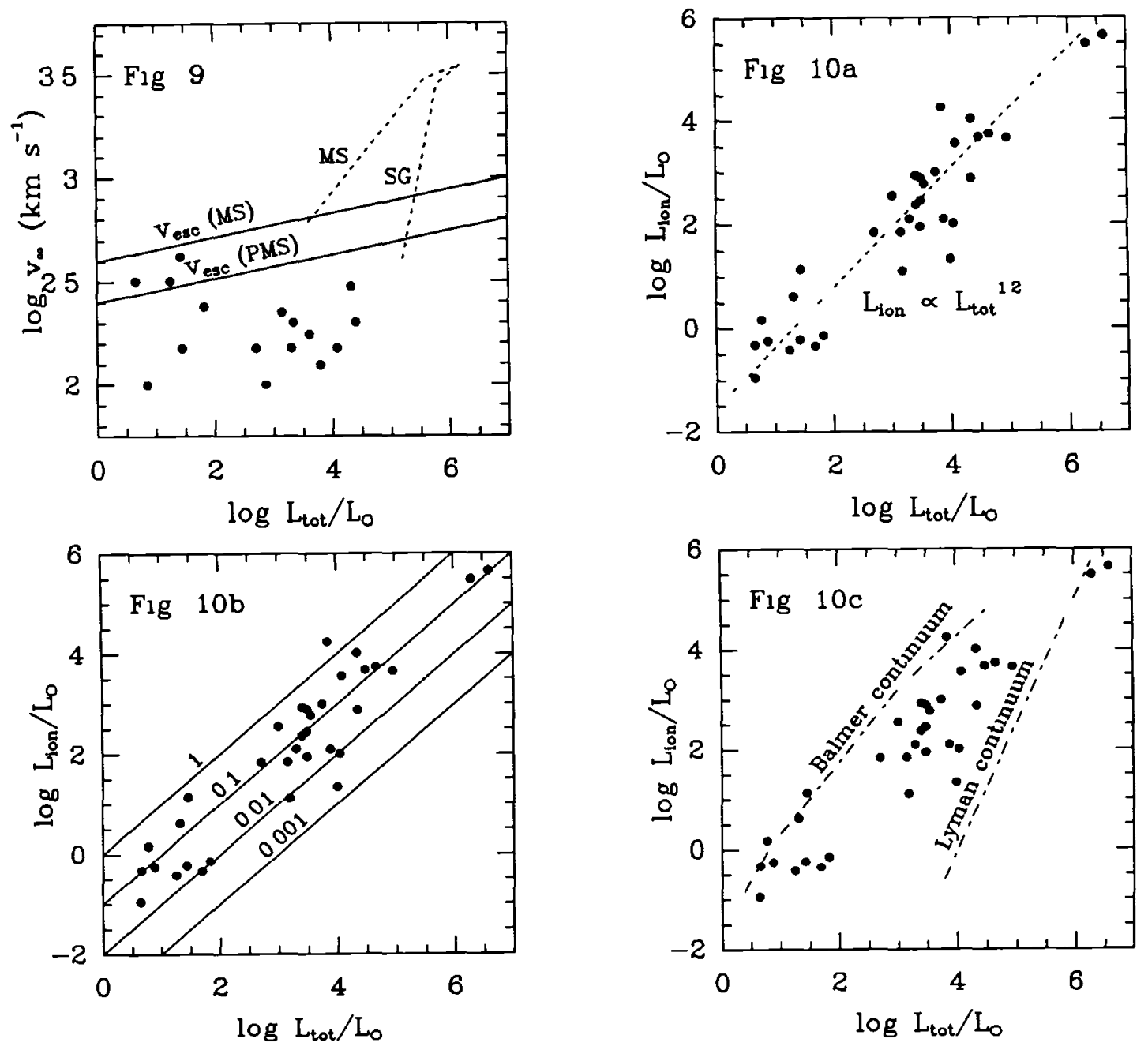

Fig. 9 - The maximum wind velocity as a function of the total luminosity. The terminal velocities for MS star and for supergiant winds are displayed. Also shown are the escape velocities curves for MS and PMS stars.

Fig. 10 - The ionization luminosity as a function of the total luminosity: a) The bestfitting power law is plotted; b) Lines of constant ratio $L_{\text {ion }} / L_{\text {tot }}$ are shown; c) The curves of the ionizing radiation in the Lyman and the Balmer continua are compared with the data.

to within a factor of three. In most cases, the ionizing flux is much higher than the stellar Lyman continuum flux but lower than the Balmer continuum flux expected on the basis of theoretical stellar atmosphere calculations (cf. Fig. 10c). Therefore, the wind could be ionized by Balmer continuum photons as suggested by Thompson (1982) and Simon et al. (1983). This problem has recently been addressed in detail by Natta et al. (1987, these proceedings). Alternatively, there may be a process which is capable to convert into ionization an energy corresponding to about $8 \%$ of the total observed stellar luminosity. Such an energy, however, cannot be provided at the expenses of the kinetic energy of the wind because this is generally smaller than $1 \%$ of the stellar luminosity (cf. Fig. 13). Among the number of possible processes the most plausible are: 

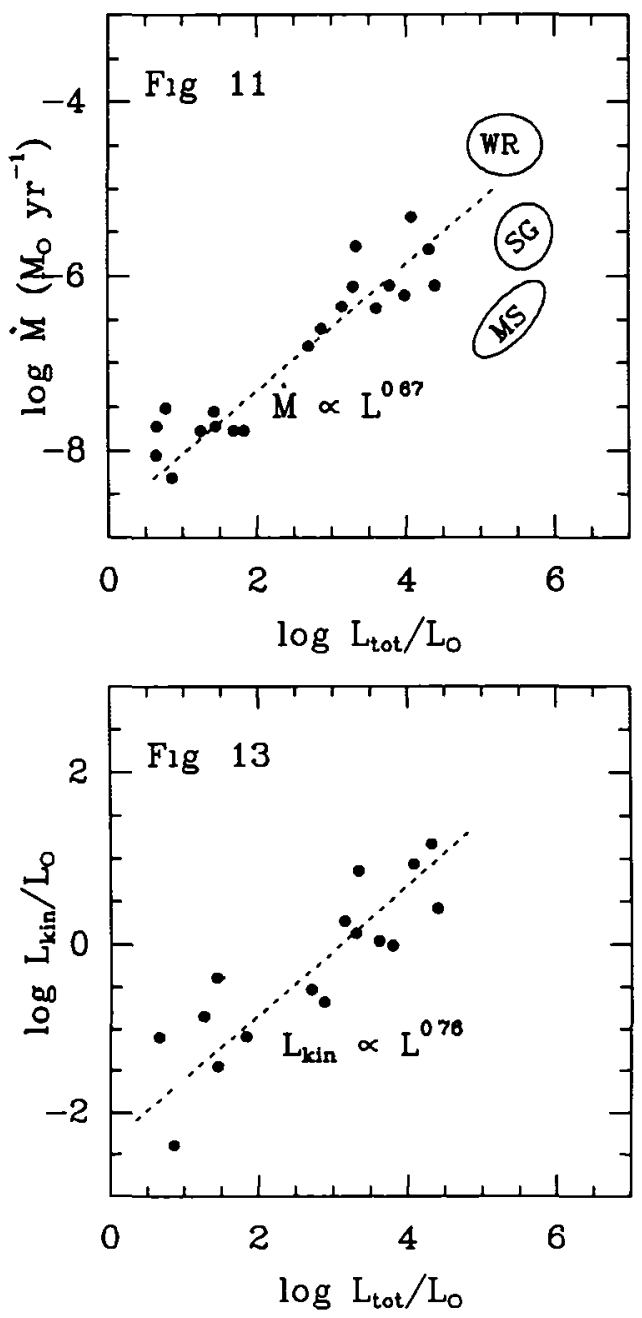

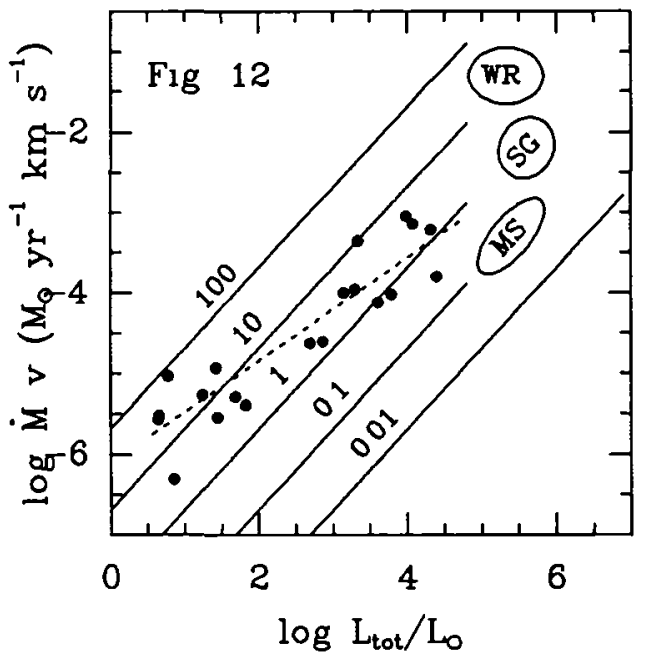

Fig. 11 - The mass loss rate as a function of the total luminosity. The best-fitting power law is shown as a straight line. The areas occupied by early type MS, supergiant (SG) and Wolf-Rayet (WR) stars are also indicated.

Fig. 12 - The momentum flux as a function of the total luminosity. The best-fitting power law is shown as a dashed line. Solid lines correspond to constant values of the ratio $(\dot{M} v) /(L / c)$. The areas of MS, SG and WR stars are also indicated.

Fig. 13 - The mechanical luminosity as a function of the total luminosity. The best-fitting power law is shown as a dashed line.

i) The presence of a hot corona at the base of the wind: its temperature should not exceed few hundred thousand, and be higher than several tens of thousand, Kelvin degrees in order to be able to provide the necessary ionizing flux but escape detection both at UV wavelengths (e.g. IUE range 1150-3200 $\AA$ ) and in the X-ray domain.

ii) Collisional ionization and excitation by thermal processes: this implies that the wind gas temperature be as high as $15000 \mathrm{~K}$ in order to make hydrogen $50 \%$ ionized. Clearly some heating agent must keep the gas at such a temperature: a possibility is dissipation of Alfven waves which have been proposed to explain the acceleration of the wind (Lago, 1984).

iii) Radiation produced by infall of matter onto the stellar surface: this accretion may represent the terminal phases of the very star formation process. In this case mass accretion and mass loss would coexist with each other: the former would prevail on and near the equatorial plane of the star and would originate from the molecular disk which gave birth to the star (Pudritz, 1985). The latter would dominate in the polar directions and, in addition to the ordinary wind manifestations, would also be responsible for the ionized component of the bipolar flows associated with PMS stars (e.g. see the discussion on $\mathrm{S} 106$ by Felli et al. 1984). 
As for the mass loss rate and the momentum flux (Figures 11 and 12) we see that again there is a good correlation of both quantities with $L_{t o t}$, of the form

$$
\begin{aligned}
& \dot{M} \propto L_{\text {tot }}^{067 \pm 004} \\
& \dot{M} v \propto L_{\text {tot }}^{068 \pm 006}
\end{aligned}
$$

The fact that the dependence of $\dot{M}$ and $\dot{M} v$ on $L_{\text {tot }}$ is virtually the same is a direct consequence of the velocity being almost independent of the luminosity. Both the mass loss rate and the momentum flux are considerably higher that what one measures in "ordinary" stars, both MS or supergiant stars (e.g. Panagia and Macchetto, 1982; Felli and Panagia, 1981) while they are comparable, after a suitable scaling, to the values observed in WR stars (e.g. Barlow et al. 1981). This indicates that stellar winds in the PMS phase are much more "active" than they will be in subsequent phases of the evolution. As a corollary, it appears that the mechanism which produces the mass loss is different from the one that operates in MS and supergiant stars but, because of the continuity of observed properties, it is likely to be the same for all PMS stars. We also see that the momentum flux $\dot{M} v$ is generally higher than the momentum carried by the radiation, $L / c$ (cf. Fig. 12). Such high values of the momentum flux demand that the wind acceleration be due to either multiple scattering of the stellar radiation or to some non-radiative process (e.g., deposition of Alfven waves: Lago 1984). The former mechanism is able to account for the wind acceleration in early type stars (Panagia and Macchetto, 1982) but in order to work with comparable efficiency for PMS stars would require a high UV luminosity: this condition would also satisfy the ionization requirements but would imply a rather peculiar spectrum in that no trace of a strong UV continuum component has been observed in PMS stars with IUE (e.g., Gahm 1980 and references therein).

Finally, an inspection of Figure 13 reveals that the mechanical luminosity appears to correlate quite well with the radiative luminosity, i e.

$$
L_{k 2 n} \propto L_{t o t}^{0.76 \pm 011}
$$

On the other hand, in all cases $L_{k \imath n}$ corresponds to a minor fraction of the total radiative luminosity

$$
L_{k 2 n} \simeq 0.01 L_{t o t}
$$

and, therefore, it cannot play any major role in the interplay star/wind/environment.

In summary we can conclude as follows:

1. One and the same mechanism appears to be responsible for the mass loss in all PMS stars. The mechanism itself, however, is not identified.

2. The wind ionization requires an agent external to the wind but the ordinary Lyman continuum radiation from the star is not enough for this purpose. Either Balmer continuum ionization or a more exotic processes is required to account for the wind ionization.

3. The acceleration of the wind is likely not to be due to radiation and may be produced by direct deposition of energy into the wind.

Finally it is worth considering the large scale properties of the winds of PMS stars and comparing them with the properties of the molecular bipolar flows which appear to be associated with PMS stars. Measurements of stellar winds probe regions of modest 
size, say less than few $10^{14} \mathrm{~cm}$, which corresponds to less than $0.1^{\prime \prime}$ at the distance of the closest $T$ associations. On the other hand, one can currently observe ordered flows much farther away from the star but which undoubtedly originate from the star itself. This is, for example, the case of DG Tau B which displays an ionized, narrow jet (opening angle less than 5 degrees; Mundt and Fried, 1983) or S106-IRS4 (see section 3.1) These PMS stars and bipolar flows or jets appear to be associated with relatively massive molecular disks. They also appear to be aligned with the molecular flows which may extend over an even larger scale and seem to arise from the molecular disks moving approximately perpendicularly to the disk itself (i.e., along the polar axis). Examples of these structures are S 106 (e.g., Felli or L 1551 (Lada, 1985 and references therein). In all cases, the momentum carried by the molecular flows is more than an order of magnitude higher than that carried by the ionized flow (Lada, 1985). Therefore, although it cannot be the stellar wind to push the molecular flow (because of the momentum problem) nor can the molecular flow directly produce the ionized flow and/or the stellar wind (because the former does not reach regions so close to the central star where the latter is observed to exist) a tight connection between the two phenomena must exist. It is quite possible that they both represent different manifestations of the same general phenomenon governing the formation of stars of any mass. 


\section{REFERENCES}

Abbott, D. C., 1980, Ap. J., 242, 1183.

Allen, D. A., and Penston, M. V., 1975, M.N.R.A.S., 172, 245

André, Ph., 1987, in "Protostars and Molecular Clouds", eds. T. Montmerle and C. Bertout (Saclay: CEA/Doc).

André, Ph., Montmerle, T, and Feigelson, E D , 1987, A. J , 93, 1182.

Bally, J. B., Snell, R. L, and Predmore, R., 1983, Ap. J., 272, 154.

Barlow, M J., Smith, L. J and Willıs, A. J., 1981, M.N R.A.S., 196, 101

Becklin, E E., and Neugebauer, G., 1967, Ap. J., 147, 799.

Bieging, J. H., Cohen, M., and Schwartz, P. R., 1984, Ap. J., 282, 699.

Brown, R. L., Broderick, J. J., and Knapp, G. R., 1976, M.N R.A.S , 175, 87 P.

Campbell, B., Persson, S. E., and McGregor, P. J., 1986, Ap. J , 305, 336

Castor, J. I., 1970, M.N.R.A.S., 149, 111.

Castor, J. I., and Simon, T., 1983, Ap. J., 265, 304.

Chiuderi, C., and Torricelli Ciamponi, G., 1978, Astr. Ap., 69, 333.

Cohen, M., and Bieging, J. H., 1986, A. J., 92, 1396.

Cohen, M., Bieging, J. H. and Schwarz, P. R., 1982, Ap. J., 253, 707.

Dulk, G. A., 1985, Ann. Rev. Astron. Astrophys., 23, 169

Evans, N. J., II, Levreault, R M., Beckwith, S. and Skrutskie, M , 1987, preprint

Feigelson, E. D., and Montmerle, T., 1985, Ap. J. (Letters), 289, L19.

Felli, M. and Panagia, N., 1981, Astr. Ap., 102, 424.

Felli, M., Gahm, G F., Harten, R., Liseau, R. and Panagia, N., 1982, Astr Ap, 107, 354.

Felli, M., et al. 1984, Astr. Ap., 135, 261.

Felli, M., Oliva, E., Stanga, R., and Panagia, N., 1985a, in "(Sub)Mıllımeter Astronomy", eds. P. Shaver and K. Kjaer, p. 503.

Felli, M., Stanga, R., Oliva, E., and Panagia, N., 1985b, Astr. Ap., 151, 27.

Felli, M., Simon, M., Fischer, J., and Hamann, F., 1985c, Astr. Ap., 145, 305.

Felli, M, and Panagia, N., 1987, in preparation.

Gahm, G. F., 1980, in "The Universe at Ultraviolet Wavelengths: The First Two Years of IUE," NASA CP-2171, p. 105.

Hamann, F., and Simon, M., 1986, Ap. J., 311, 909.

Hughes, V. A., 1985, Ap. J., 298, 830.

Krolik, J. H., and Smith, H A., 1981, Ap. J., 249, 628.

Lada, C. J., 1985, Ann. Rev. Astron. Astrophys., 23, 267.

Lada, C J., 1986, in "Star Forming Regions", eds. M. Peimbert and J. Jugaku (Dordrecht. Reidel), p. 1.

Lada, C. J., and Wilking, B. A., 1984, Ap. J., 287, 610

Lago, M. T. V. T., 1984, M.N.R.A.S., 210, 323

Lamers, H. J. G. L. M., and Waters, L. B. F M., 1984, Astr. Ap., 136, 37

Marsh, K. A., 1975, Ap. J., 201, 190.

Mezger, P. G., and Henderson, A. P., 1967, Ap. J., 147, 471.

Moran, J. M., Garay, G., Reid, M. J., Genzel, R , Wright, M. C. H. and Plambeck, R. L., 1983, Ap. J. (Letters), 271, L31.

Mundt, R. and Fried, J. W., 1983, Ap. J. (Letters), 274, L83. 
Natta, A., Palla, F, and Giovanardi, C., 1987, these proceedings.

Olnon, F., 1975, Astr. Ap., 39, 217.

Panagia, N., 1985, Physica Scripta, T11, 71.

Panagia, N. and Felli, M., 1975, Astr. Ap., 39, 1.

Panagia, N. and Macchetto, F., 1982, Astr. Ap., 106, 226.

Panagia, N., Oliva, E., and Felli, M., 1987, in preparation.

Persson, S. E., Geballe, T. R., McGregor, P. J., Edwards, S. and Lonsdale C. J., 1984, Ap. J., 286, 289.

Persson, S. E., McGregor, P. J., and Campbell, B., 1987, preprint.

Pudritz, R. E., 1985, Ap. J., 293, 216.

Reynolds, S. P., 1986, Ap. J., 304, 713.

Rodriguez, L. F., and Cantó, J., 1983, Rev. Mexicana Astron. Astrof., 8, 163.

Rydgren, A. E., Schmelz, J. T., Zak, D. S., and Vrba, F. J., 1984, Pub. U.S. Naval Obs., Second Series, Vol. XXV, Part I.

Schmid-Burgk, J., 1982, Astr. Ap., 108, 169.

Sibille, F., Bergeat, J., Lunel, M., and Kandel, R., 1975, Astr. Ap., 40, 441.

Simon, M., Felli, M., Cassar, L., Fischer, J. and Massi, M., 1983, Ap. J., 266, 623.

Simon, M., and Fischer, J., 1982, B.A.A.S., 14, 925.

Smith, H. A., Fischer, J., Geballe, T. R., and Schwartz, P. R., 1987, Ap. J., 316, 265.

Snell, R. L., and Bally, J. B., 1986, Ap. J., 303, 683.

Sobolev, V. V., 1958, in "Theoretical Astrophysics", ed. V. A. Ambartsumyan (New York: Pergamon Press), p. 482.

Tanzi, E. G., Tarenghi, M., and Panagia, N., 1981, in "Effects of Mass Loss on Stellar Evolution", eds. C. Chiosi and R. Stalio, (Dordrecht: Reidel), p. 51.

Thompson, R. I., 1982, Ap. J., 257, 171.

Thompson, R. I., 1987, Ap. J., 312, 784.

Thompson, R. I., Thronson, H. A. and Campbell, B., 1983, Ap. J., 266, 614.

White, R. L., 1985, Ap. J., 289, 698.

Wilking, B. A., and Lada, C. J., 1983, Ap. J., 274, 698.

Wilking, B. A., Mundy, L. G., and Schwartz, R. D., 1986, Ap. J. (Letters), 303, L61.

Wright, A. E., and Barlow, M. J., 1975, M.N.R.A.S., 170, 41. 
End of Document 